\title{
A Rare Presentation of Orbital Roof Blow-out Fracture with Intact Vision
}

\author{
Arbindra Kumar Yadav ${ }^{1}$, Manohar Kumar Ray ${ }^{1}$, Birat Thapa Magar ${ }^{1}$, Dinesh \\ Kumar Thapa $^{1}$, Karuna Tamrakar Karki ${ }^{1}$, Pankaj Raj Nepal ${ }^{1}$. \\ ${ }^{1}$ Department of Neurosurgery, B \& C Medical College and Teaching Hospital \& \\ Research Center,Birtamode,Jhapa,Nepal \\ Correspondence: \\ Dr Arbindra Kumar Yadav. \\ Department of Neurosurgery, B \& C Medical College and Teaching Hospital \& \\ Research Center,Birtamode,Jhapa,Nepal. \\ Email: arbindrayadav@yahoo.com \\ Phone: +9779814701254
}

The orbital blowout fracture is most common in its inferior and medial wall; however we are presenting here a rare case of superior orbital wall blowout fracture with intact vision. This is a presentation of a 55 year-old right handed gentleman following road traffic accident had left orbital injury causing orbital roof fracture. Despite of such a huge injury there was no deterioration of his vision and his movements of the eye balls were also absolutely preserved.

Key words: Orbital blow out fracture, facial injuries, orbital roof fracture,

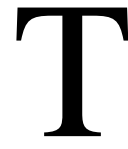
he orbital blowout fracture is most common in the base that is inferior wall blowout. We are presenting a rare case of superior orbital wall blowout fracture with preservation of vision and movement of eye ball.

Case presentation: A 55 year-old right handed gentleman was brought to emergency department in our hospital with alleged history of road traffic accident with laceration wound over left supraorbital region $(5 \times 2 \mathrm{~cm})$ and right occipital region $(7 \times 2 \mathrm{~cm})$, and bilateral raccoon eyes, the darkening of the raccoon was more on the left side. He had brief history of loss of consciousness, however had no seizure

32

Date Submitted: 23/7/2019

Date Accepted: 2/9/2019 following trauma. On examination visions were intact, extraocular muscles were in full range motion and there was no diplopia. CT scan of head revealed left superior orbital wall blowout fracture with underlying pneumocephalus and small contusion. He was managed conservatively for head injury including the orbital trauma. Debridement and suturing was done for lacerated wound. During the course of his hospital stay there was no deterioration of his vision or any neurological functions.

egneuro Volume 01, Issue 02, 2019

DOI: https://doi.org/10.3126/egn.v1i2.25732 


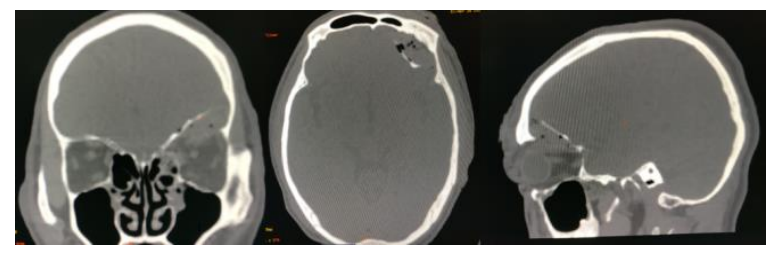

Figure 1: Bone window showing coronal, axial and sagittal images of the patient evidence of left orbital roof fracture.

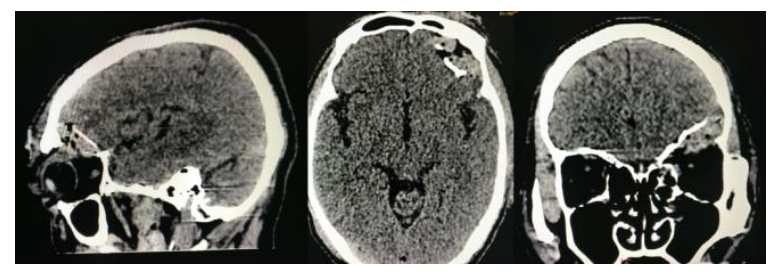

Figure 2: Brain window showing sagittal, axial, coronal images of the patient evident of left orbital roof fracture and pneumocephalus.

Discussion: Orbital blowout fracture are more common on the inferior and the medial wall of the orbit because of its thin architecture. They usually present with double vision, sunken ocular globe, numbness of cheek and upper gums due to injury to the infraorbital nerve. ${ }^{1}$ Broadly, this type of fractures are classified as open door and trap door and occasionally associated with other injuries as Le Forte fractures or zygomaticomaxillary complex farctures ${ }^{2}$. However, here we have presented a case of the isolated orbital roof fracture with intact orbital rim, which was secondary to road traffic accident which is the commonest mode of such injuries.

The name blowout is secondary to its sudden raise of intraorbital pressure causing the herniation of the orbital contents. ${ }^{3}$ The other theory of inferior orbital wall fracture is cranial shift due to raised intracranial pressure. $^{4}$

All types of orbital blow out fractures can lead to complications of orbital rim displacement loss of vision, diplopia, exophthalmus/enophthalmus due to raised intraorbital pressure or entrapment of soft tissue can occur which most commonly require surgery to release and sometimes requires orbit roof reconstruction surgery. ${ }^{5}$

The isolated superior orbital roof blowout fracture is defined as superior displacement of the fracture fragment into the anterior cranial fossa without involvement of supraorbital rim, with possible herniation of orbital contents outside of the orbital confines. The most commom mechanism of superior orbital roof fracture is high energy blunt trauma to the orbit or forehead. Very rarely orbital roof fracture occurs without displacement of the orbital rim, also called undisplaced orbital roof fracture.

Conclusion: Though orbital blowout fractures are more common in the inferior and the medial wall of the orbit, isolated orbital roof fracture is also possible as evident in this case.

\section{Conflict of interest: None.}

\section{Reference:}

1. Caranci, F; Cicala, D; Cappabianca, S; Briganti, F; Brunese, L; Fonio, P (2012). "Orbital fractures: Role of imaging". Seminars in Ultrasound, CT and MRI. 33 (5): 385-91.

2. Ellis e, 3rd (2012). "Orbital trauma". Oral and Maxillofacial Surgery Clinics of North America. 24 (4): 629 48. doi:10.1016/j.coms.2012.07.006.

3. Smith B, Regan WF Sr. Blowout fracture of the orbit: mechanism and correction of internal orbital fractures . Am J Ophthalmo/1957;44 :733-739

4. Jones A.L., Jones K.E. Orbital roof "blow-in" fracture: a case report and review. J. Radiol. Case Rep. 2009;3(12):25-30.

5. Jones DEP, Evans JNG. " Blowout" fractures of the orbit: an investigation into their anatomical basis. J Laryngol Otol 1967;8 $1: 1109-1120$ 